\title{
No-reference image quality assessment based on AdaBoost_BP neural network in wavelet domain
}

\author{
YAN Junhua ${ }^{1,2, *}$, BAI Xuehan ${ }^{1}$, ZHANG Wanyi ${ }^{1}$, XIAO Yongqi $^{1}$, CHATWIN Chris ${ }^{2}$, \\ YOUNG Rupert ${ }^{2}$, BIRCH Phil ${ }^{2}$ \\ 1. College of Astronautics, Nanjing University of Aeronautics and Astronautics, Nanjing 210016, China; \\ 2. School of Engineering and Informatics, University of Sussex, Brighton BN1 9QT, UK
}

\begin{abstract}
Considering the relatively poor robustness of quality scores for different types of distortion and the lack of mechanism for determining distortion types, a no-reference image quality assessment (NR-IQA) method based on the AdaBoost_BP neural network in the wavelet domain (WABNN) is proposed. A 36dimensional image feature vector is constructed by extracting natural scene statistics (NSS) features and local information entropy features of the distorted image wavelet sub-band coefficients in three scales. The ABNN classifier is obtained by learning the relationship between image features and distortion types. The ABNN scorer is obtained by learning the relationship between image features and image quality scores. A series of contrast experiments are carried out in the laboratory of image and video engineering (LIVE) database and TID2013 database. Experimental results show the high accuracy of the distinguishing distortion type, the high consistency with subjective scores and the high robustness of the method for distorted images. Experiment results also show the independence of the database and the relatively high operation efficiency of this method.
\end{abstract}

Keywords: image quality assessment (IQA), AdaBoost_BP neural network (ABNN), wavelet transform, natural scene statistics (NSS), local information entropy.

DOI: 10.21629/JSEE.2019.02.01

\section{Introduction}

High quality natural images with rich information can help ensure the accuracy and effectiveness of the information transmission, which can enhance the ability of the perceived image information. The quality of images can be affected by various factors in the imaging procedure, such as image acquisition, transmission and processing; thus it is crucial to quantify the effects of various distortions on

Manuscript received December 19, 2017

*Corresponding author.

This work was supported by the National Natural Science Foundation of China (61471194; 61705104), the Science and Technology on Avionics Integration Laboratory and Aeronautical Science Foundation of China (20155552050), and the Natural Science Foundation of Jiangsu Province (BK20170804). image quality [1]. The no-reference image quality assessment (NR-IQA) method can assess the distorted images without the information of perfect reference images. Moreover, human observers can assess the quality as well as the distortion type without the reference images. Considering the perfect reference images are usually unavailable in practice, the NR-IQA has high values in the research of the human visual system (HVS) as well as the image quality assessment and practical applications.

NR-IQA methods are usually divided into two categories. One is for a specific type of distortion. Zhu et al. [2] proposed a method for white noise (WN) images utilizing the local gradient feature. Chen et al. [3] and Marichal et al. [4] proposed a method for blur images by using multi-scale gradient features and histogram of non-zero discrete cosine transform (DCT) coefficients. Shao et al. [5] proposed a method for blur and noise images based on the HVS and the structural similarity. Wang et al. [6] proposed a method for joint photographic experts group (JPEG) images by using block effects. Sazzad et al. [7] proposed a method by using the pixel and edge information for joint photographic experts group 2000 (JP2K) distorted images. Horita et al. [8] proposed a method based on the blackness, the average absolute difference and the zero-crossing rate for JPEG/JP2K. Xie et al. [9] proposed a method for images distorted by haze based on the contrast map calculated by Weber's law. Those methods need to know the type of image distortion and are limited when applied in practice [10]. The other category is to establish a general NR-IQA model. Most of these models rely on machine learning such as support vector machine (SVM), neural network, etc. Moorthy et al. [11] proposed a method in the two-step framework of the blind image quality index (BIQI). The 18-dimensional natural scene statistics (NSS) features are extracted first, then the SVM classification is used to calculate the probability that the image belongs to each distortion type, then the support vector 
regression (SVR) analysis model is used to calculate the quality score of the distorted images belonging to different types of distortion, and the final score is obtained according to the probability weighting. Li et al. [12] proposed an NR-IQA method by using the generalized regression neural network (GRNN). The phase congruency and gradient features of the images are extracted, and the mapping relation between the features and the image quality is simulated by the neural network to achieve the quality assessment. The real-time performance of the BIQI and the GRNN is relatively poor due to the complexity of parameter setting and model mapping. Thus, some scholars work on improving the BIQI method. Moorthy et al. [13] proposed the distortion identification-based image verity and integrity evaluation (DIIVINE) with more 88-dimensional NSS features in the wavelet domain. The performance of the DIIVINE is better for the WN distortion; for other types, the performance is only average. Saad et al. $[14,15]$ proposed the blind image integrity notate using DCT statistics (BLIINDS-II), which extracts the NSS features in the DCT domain. The BLIINDS-II performs well, but it is of high computation complexity. Mittal et al. [16-18] proposed the natural image quality evaluation (NIQE) method and the blind/referenceless image spatial quality evaluation (BRISQUE) method, both of which extract the NSS features in the spatial domain. Liu et al. [19] proposed the spatial-spectral entropy based quality index (SSEQ) method, which extracts spatial entropy features and spectral entropy features. The performance of the SSEQ for WN distorted images needs to be improved. Li et al. [20] proposed the blind IQA metric based on high order derivatives (BHODs) algorithm, using the histogram features of local binary patter (LBP) corresponding to the original map and the 3-order gradient map to evaluate image quality. The BHOD has a high subjective consistency. However, the number of the features is too big. Li et al. [21] proposed the no-reference quality assessment using statistical structural and luminance features (NRSL), which extracts local normalized luminance features and LBP features of the local normalized luminance map to build the NR model. The NRSL has a high subjective consistency. However, the transfer-domain features are not extracted. Liu et al. [22] proposed the oriented gradients IQA (OGIQA), which extracts the gradient feature and uses the AdaBoosting_BP to obtain the quality score. The NRSL and the OGIQA perform well. However, the transfer-domain features are not extracted. Most of the existing NR-IQA methods can only calculate the quality score of the distorted image, and they cannot produce the judgment of the image distortion type, so the practical applicability is limited.
Considering the relatively poor robustness of quality scores for different types of distortions and the lack of mechanism for determining distortion types, an NR-IQA method based on the adaBoost_bP neural network in the wavelet domain (WABNN) is proposed. A 36-dimensional image feature vector is constructed by extracting the NSS features and the local information entropy features of the distorted image wavelet sub-band coefficients in three scales. The ABNN scorers and classifiers are trained to obtain an image quality score and determine the type of the image distortion.

\section{Image feature extraction in wavelet domain}

High quality natural images have regular statistical characteristics, and the distortions can alter the image structure as well as the statistical characteristics [23,24]. Thus, the type and degree of the distortion can be characterized by the changes of the statistical characteristics. The distortion can alter the image information entropy. Therefore, the type and degree of the distortion can also be characterized by the changes of the information entropy. Moreover, considering the multi-channel characteristics of human eyes, the proposed method extracts 18 -dimensional NSS features $\boldsymbol{F}_{1}$ and 18-dimensional local information entropy features $\boldsymbol{F}_{2}$ in the wavelet domain to construct a 36-dimensional image feature vector $\boldsymbol{F}=\left(\boldsymbol{F}_{1}, \boldsymbol{F}_{2}\right)^{\mathrm{T}}$.

\subsection{Image NSS features in the wavelet domain}

\subsubsection{Determination of image distortion type based on wavelet subband coefficient distribution}

There are certain statistical laws in natural images, which are closely related to the image quality and will change with different distortions. As an example shown in Fig. 1, with different mean opinion scores (DMOS) a reference (REF) image and the corresponding five distorted images of different distortion types are randomly selected from the laboratory of image and video engineering (LIVE) database [25-27]. The distortion types include Gaussian WN, Gaussian blur (BLUR), fast rayleigh fading (FF), JPEG and JP2K. Fig. 2 shows the first scale normalized histograms of wavelet sub-band coefficients (WSCs) for the images of Fig. 1 in diagonal orientation.

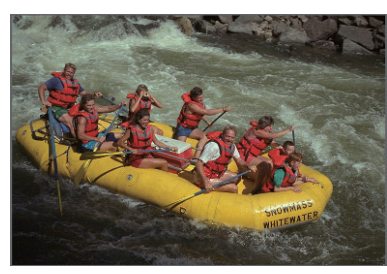

(a) REF DMOS $=0$

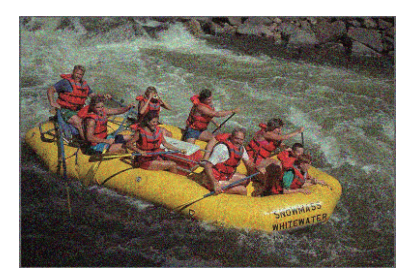

(b) $\mathrm{WN}$ DMOS $=54.62$ 


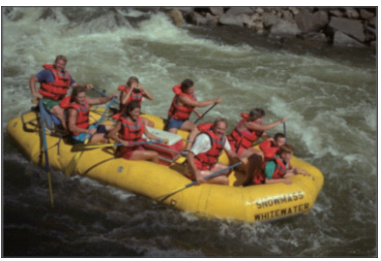

(c) BLUR DMOS=56.67

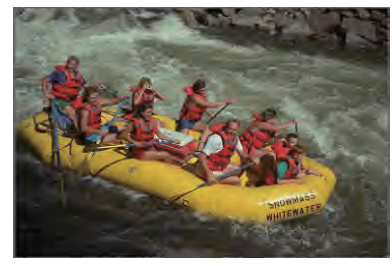

(e) JP2K DMOS=76.28

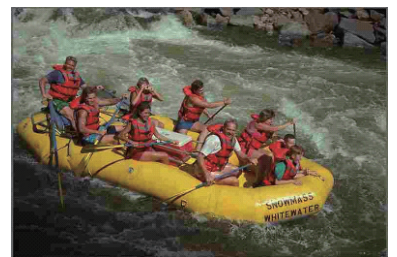

(d) JPEG DMOS=59.74

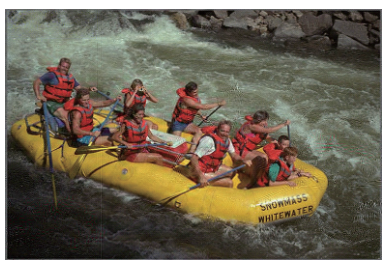

(f) FF DMOS=57.01
Fig. 1 REF image and the five corresponding $\mathrm{WN}$ distorted images with different distortion degrees

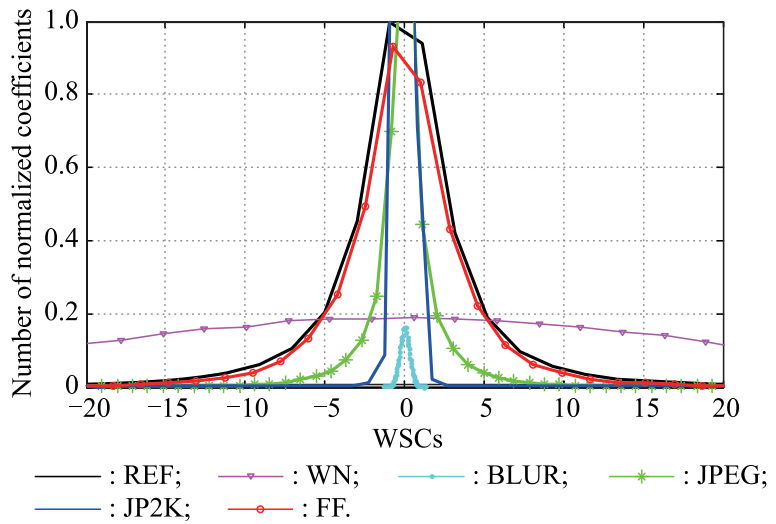

Fig. 2 First-scale normalized histograms of WSC for the images of Fig. 1 in diagonal orientation

Fig. 2 shows that the histogram distributions of WSCs are zero means, and different from the different types of distortions. The WN image has a relatively flat coefficient distribution curve, indicating that the WSC distribution is relatively uniform. The distribution curve of the FF is most similar to and basically in coincidence with that of the REF image. The coefficient distribution curves of the JPEG image and the JP2K image are similar, but the curve of the JP2K image is sharper, and the number of sub-band coefficients at mean is larger than that of the REF image. The distribution of the BLUR is the most concentrated, with fewer coefficients around the mean. The WN, FF, BLUR, JPEG and JP2K distortion can be distinguished by histogram distributions of WSCs. Thus, the distribution of the WSCs can be used as an indicator of the distortion type.

\subsubsection{Determination of image distortion degree based on the WSC distribution}

As shown in Fig. 3, taking WN as an example, a REF image and the corresponding five distorted images with different degrees of distortion are randomly taken from the
LIVE to explore the relationship between the WSC distribution and the degree of distortions.

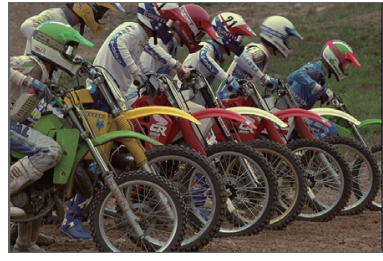

(a) REF

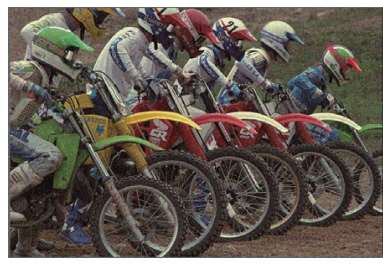

(c) WN DMOS $=45.82$

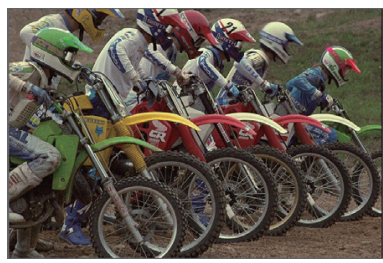

(e) WN DMOS $=23.51$

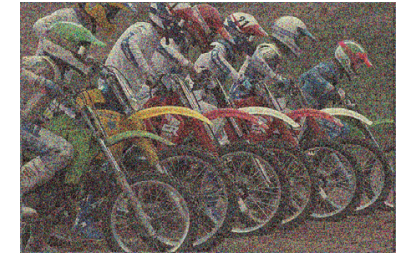

(b) $\mathrm{WN}$ DMOS $=74.63$

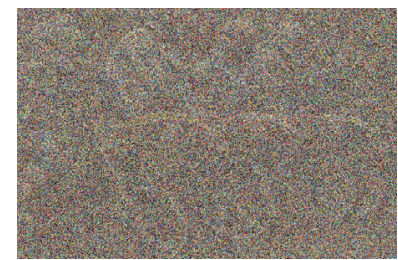

(d) WN DMOS $=105.10$

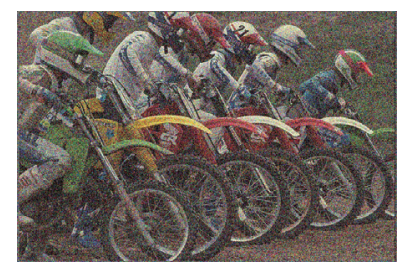

(f) WN DMOS $=65.71$
Fig. 3 REF image and the corresponding five WN distorted images with different distortion degrees

The wavelet transform over three scales and three orientations is also applied to these six images, and Fig. 3(a) is taken as the example shown in Fig. 4.

The wavelet sub-band of Fig. 3 for the first scale and the horizontal orientation is taken as the example as shown in Fig. 5.

The first scale normalized histogram of WSCs for the images of Fig. 5 in horizontal orientation is shown in Fig. 6. The higher the DMOS value is, the smoother the distribution curve is. Overall, the peak value of curves at zero is lower than that of the REF image. Thus, the distribution of the WSC can be used as an indicator of the distortion degree.

\subsubsection{Extracting image NSS features in the wavelet domain}

A wavelet sub-band of an image contains thousands of coefficients. The total number of all sub-bands coefficients is very large. Considering the computational complexity and efficiency, in the current method, the statistical model, instead of the WSC, is used to characterize the image features. The edge distribution [11] of the WSC for a natural image accords with a generalized Gaussian distribution (GGD) model. 


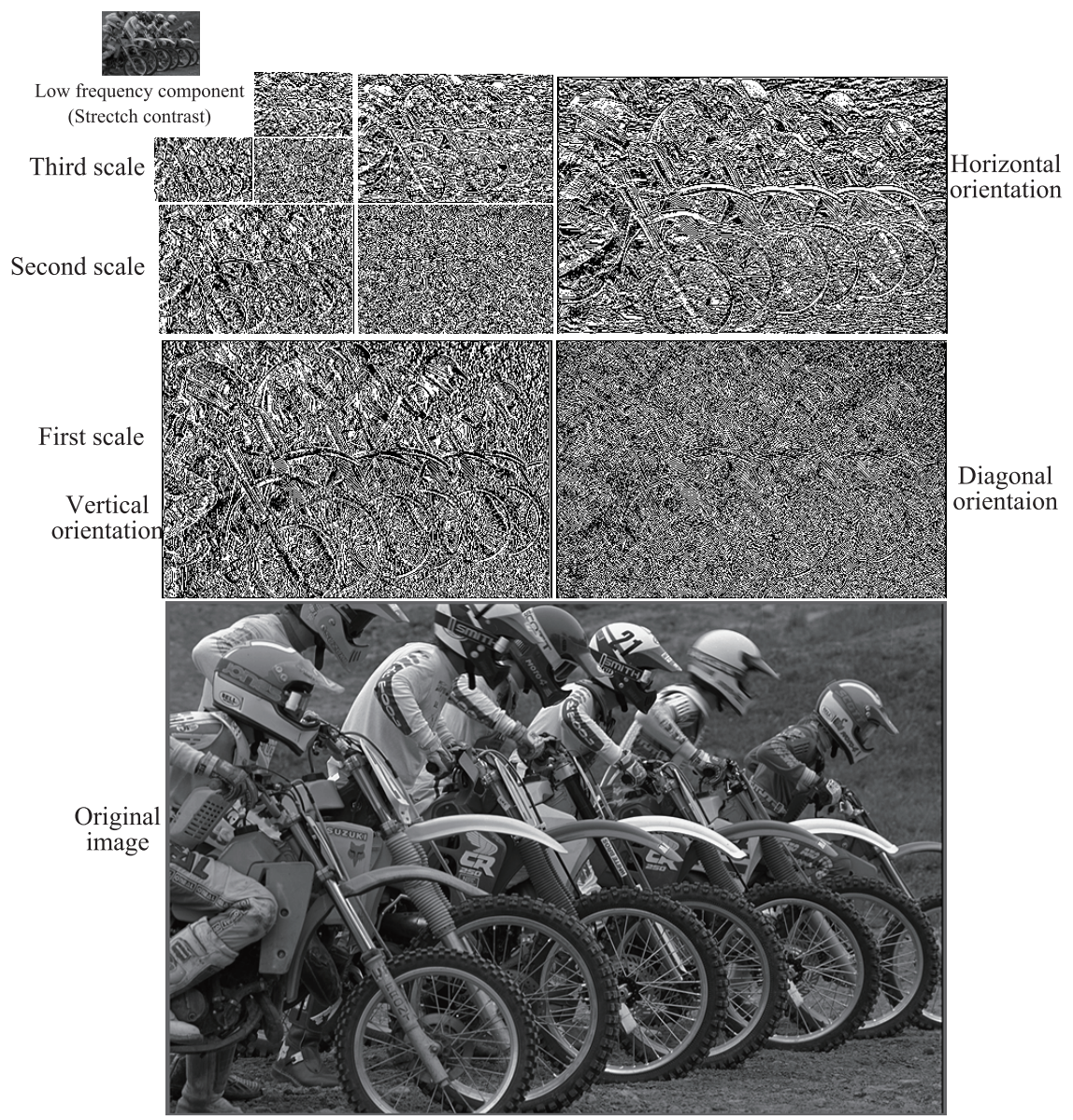

Fig. 4 Wavelet transform over three scales and three orientations on Fig. 3(a)

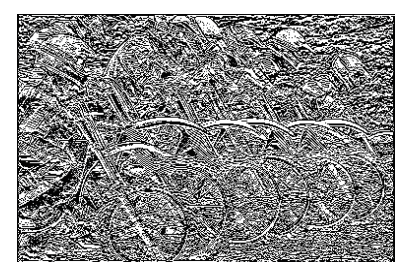

(a) REF

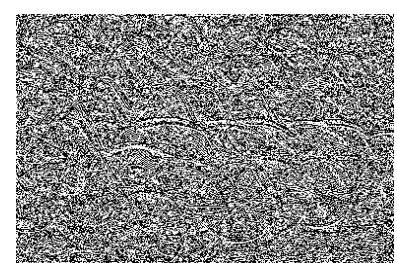

(c) WN DMOS $=45.82$

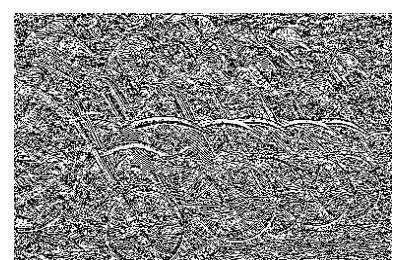

(e) WN DMOS $=23.51$

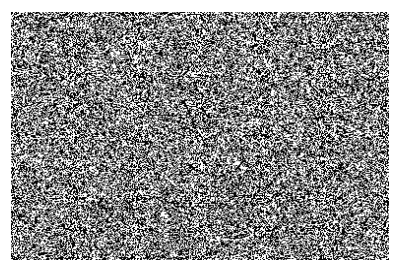

(b) WN DMOS $=74.63$

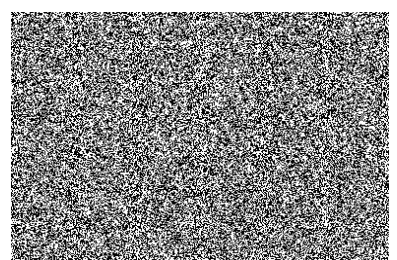

(d) WN DMOS $=105.10$

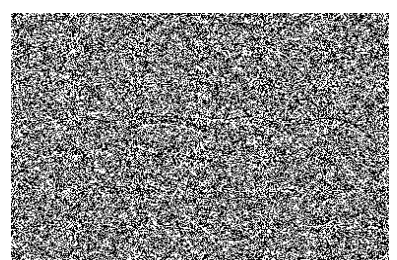

(f) WN DMOS=65.71

Fig. 5 Wavelet sub-band of Fig. 3 for the first scale and horizontal orientation

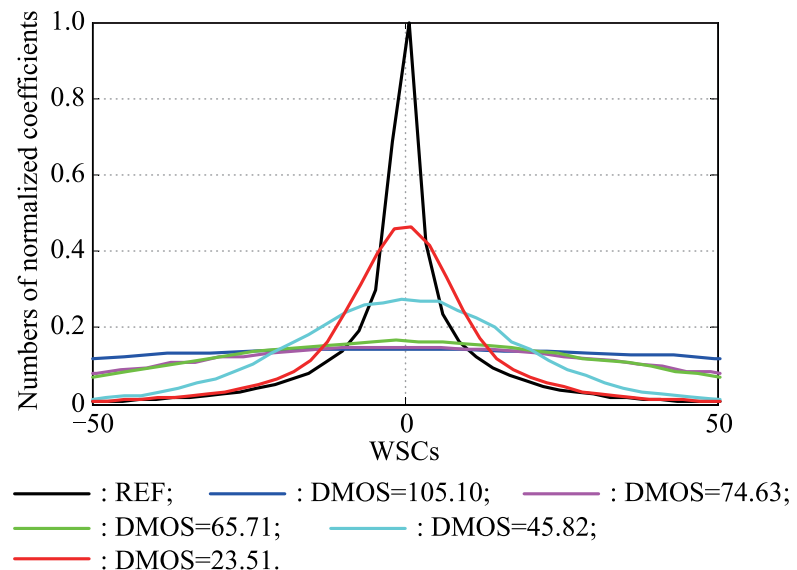

Fig. 6 First scale normalized histograms of WSCs for the images of Fig. 5 in horizontal orientation

The GGD model is defined as follows:

$$
g_{X}\left(x ; \mu, \sigma^{2}, \gamma\right)=\alpha \exp \left(-[\beta|x-\mu|]^{\gamma}\right), \quad x \in \Re
$$

where $x$ is a wavelet sub-band of the image, $\mu$ is the mean of the coefficients on the sub-band $x, \sigma^{2}$ is the variance of the coefficients on the sub-band $x, \gamma$ is the shape parameter of the GGD model fitted by the sub-band $x$. $\alpha$ and $\beta$ are proportional constants and are defined as follows: 


$$
\left\{\begin{array}{l}
\alpha=\frac{\beta \gamma}{2 \Gamma(1 / \gamma)} \\
\beta=\frac{1}{\sigma} \sqrt{\frac{\Gamma(3 / \gamma)}{\Gamma(1 / \gamma)}}
\end{array}\right.
$$

where $\Gamma(\cdot)$ is a function defined as follows:

$$
\Gamma(z)=\int_{0}^{\infty} t^{z-1} \mathrm{e}^{-t} \mathrm{~d} t, \quad z>0 .
$$

The three parameters $\mu, \sigma^{2}$ and $\gamma$ of the GGD model are obtained by using the method of the generalized Gaussian proportional function [28].

Since the wavelet transform decomposes the image into multiple-channels, which is similar to the bandpass filter, it

can be assumed that the mean of the WSC of the REF image and different types distorted images are all zero, that is, $\mu=0$, as shown in Fig. 2 and Fig. 6. In this case, the GGD model, which represents the distribution of the subband $x$, contains only two parameters $\sigma^{2}$ and $\gamma$. Therefore, a two-parameter GGD model is used to fit the coefficient distribution of a wavelet sub-band. In this way, different from the original expression which needs thousands of sub-band coefficients, the expression of image features (distortion type and degree) can be simplified to two parameters. For a distorted image, 18 wavelet-domain NSS parameters can be extracted in three scales and three orientations. The 18-dimensional vector consists of 18 parameters, that is $\boldsymbol{F}_{1}=\left(f_{1}, f_{2}, \ldots, f_{18}\right)$. The meanings of the elements in this 18-dimension vector are shown in Table 1.

Table 1 Meaning of image NSS feature vector elements in wavelet domain

\begin{tabular}{cc}
\hline Vector elements & Meaning \\
\hline$f_{1}-f_{3}$ & Variance $\sigma^{2}$ of the three sub-bands in the horizontal orientations and the first, second and third scales \\
$f_{4}-f_{6}$ & Variance $\sigma^{2}$ of the three sub-bands in the vertical orientations and the first, second and third scales \\
$f_{7}-f_{9}$ & Variance $\sigma^{2}$ of the three sub-bands in the diagonal orientations and the first, second and third scales \\
$f_{10}-f_{12}$ & Shape parameter $\gamma$ of the three sub-bands in the horizontal orientations and the first, second and third scales \\
$f_{13}-f_{15}$ & Shape parameter $\gamma$ of the three sub-bands in the vertical orientations and the first, second and third scales \\
$f_{16}-f_{18}$ & Shape parameter $\gamma$ of the three sub-bands in the diagonal orientations and the first, second and third scales \\
\hline
\end{tabular}

\subsection{Image information entropy feature in the wavelet domain}

\subsubsection{Image two-dimensional local information entropy in the wavelet domain}

For an image $A$, the pixel can be seen as a random variable, and the image information entropy can represent the average information quantity. The image information entropy can be expressed as follows:

$$
H=-\sum_{a \in A} p(a) \log _{2} p(a)
$$

where $p(a)$ is the probability of a pixel with a gray value of $a$ in the image.

The image information entropy, also known as onedimensional entropy, represents the aggregation feature of the gray distribution in the image. However, there are block structures for the distribution of the natural image, and there are correlations between the pixels in geometric positions. The one-dimensional entropy cannot well reflect the spatial characteristics of the image. Therefore, the twodimensional information entropy is used to represent the structural information of the image [29].

The local entropy of the image can better reflect the distortion comparing with the global entropy. In this paper, based on the wavelet decomposition, coefficients of a wavelet sub-band are divided into blocks and each block is named as the unit block $W$. The size of $W$ is $8 \times 8$ and the amount of $W$ is $M \times N$. The WSC of $W$ is normalized to obtain the spectral probability of $W$ in the wavelet domain:

$$
P(i, j)=\frac{w(i, j)^{2}}{\|\boldsymbol{W}\|_{2}^{2}+C}
$$

where $w(i, j)$ is the WSC in the unit block $\boldsymbol{W}, 1 \leqslant i \leqslant 8$, $1 \leqslant j \leqslant 8,\|W\|_{2}$ is the two-norm of matrix $\boldsymbol{W}$, and $C$ is a minimal positive constant and set to be $10^{-7}$.

$H_{w}(m, n)$, the wavelet-domain information entropy of $\boldsymbol{W}$, can be expressed as follows:

$$
H_{w}(m, n)=-\sum_{i} \sum_{j} P(i, j) \log _{2} P(i, j)
$$

where $m=1,2, \ldots, M, n=1,2, \ldots, N$.

The wavelet-domain 2-dimensional local information entropy $\boldsymbol{H}_{w}$ is constructed by computing the information entropy $H_{w}(m, n)$ of all $M \times N$ blocks in a wavelet subband.

2.2.2 Determination of the type and degree of image distortion based on image two-dimensional local information entropy

The normalized histogram of the two-dimensional local information entropy of the sub-band in the first scale and diagonal orientation for images in Fig. 1 is shown in Fig. 7. 
Fig. 7 shows that the mean value of the wavelet sub-band local information entropy for the reference image is about 7 , and thus the skewness is left skewed (central mean value is 8). The mean and the skewness of the curve vary with different types of distortions. For WN, BLUR and JP2K, the mean of the curve increases, while the mean of JPEG and FF decreases. As for skewness, JP2K results in an obvious right skew condition. The image wavelet sub-band local information entropy varies with different types of distortion. Thus, the local information entropy can be used to distinguish different types of image distortion.

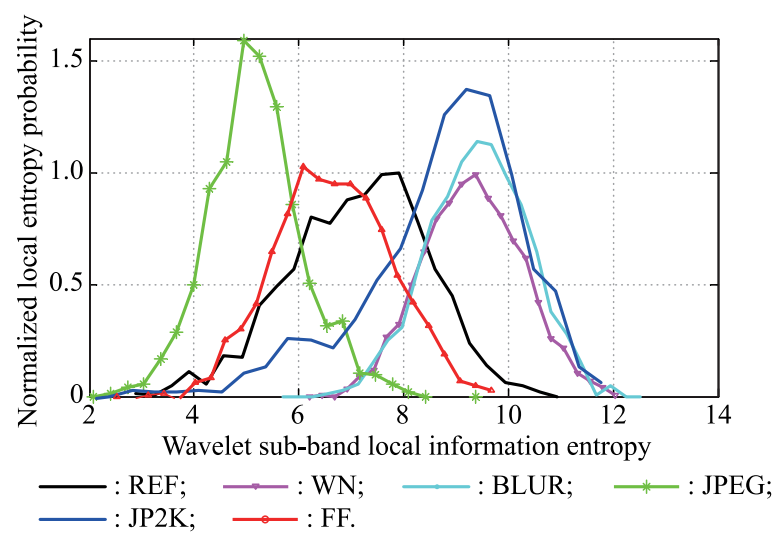

Fig. 7 Normalized histogram of two-dimensional local information entropy of the sub-band in the first scale and diagonal orientation for images in Fig. 1

Fig. 8 is the normalized histogram of the twodimensional local information entropy of the sub-bands in the first scale and diagonal orientation for images in Fig. 3. The higher the DMOS value is, the larger the mean of wavelet sub-band local information entropy is. The mean value varies with the degree of distortion and can effectively distinguish the degree of distortion. Thus, the mean value of the local information entropy can be used to distinguish different degrees of the image distortion.

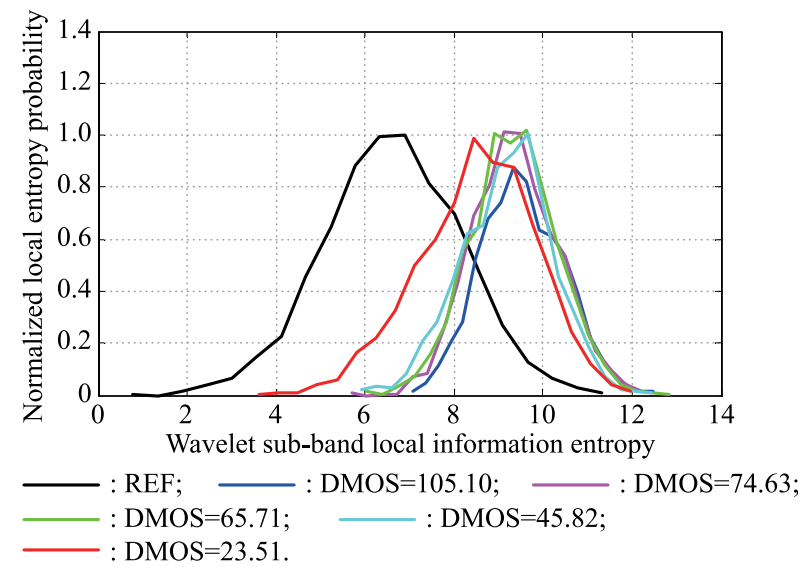

Fig. 8 Normalized histogram of two-dimensional local information entropy of the sub-band in the first scale and diagonal orientation for images in Fig. 3
In conclusion, the wavelet sub-band local information entropy can be used to distinguish the type and degree of the image distortion.

\subsubsection{Features extraction of two-dimensional local information entropy in the wavelet domain}

For a distorted image, the two-dimensional local information entropy $\boldsymbol{H}_{w}$ is a matrix whose size is $M \times N$. It contains $M \times N$ parameters, which is not suitable as image features. In this paper, two parameters of $\boldsymbol{H}_{w}$, mean and skewness, are selected as the local information entropy features of the wavelet sub-band. The mean $\mu_{w}$ and skewness $S_{w}$ are defined as follows:

$$
\begin{aligned}
& \mu_{w}=\frac{\sum_{m=1}^{M} \sum_{n=1}^{N} H_{w}(m, n)}{M \times N} \\
& S_{w}=\frac{(M \times N)^{2}}{(M \times N-1)(M \times N-2)} \times \\
& \frac{1}{M \times N} \sum_{m=1}^{M} \sum_{n=1}^{N}\left[H_{w}(m, n)-\mu_{w}\right]^{3} \\
& \sqrt{\left(\sqrt{\frac{1}{M \times N-1} \sum_{m=1}^{M} \sum_{n=1}^{N}\left[H_{w}(m, n)-\mu_{w}\right]^{2}}\right)^{3}}
\end{aligned}
$$

For a distorted image, 18 parameters can be extracted from nine sub-bands which are obtained by three scales and three orientations wavelet decomposition. The 18dimensional vector consists of 18 parameters, that is $\boldsymbol{F}_{2}=$ $\left(f_{19}, f_{20}, \ldots, f_{36}\right)$. The meanings of the elements in this 18-dimension vector are shown in Table 2.

\section{Method of NR-IQA based on WABNN}

The NR-IQA method based on the WABNN is proposed. This method extracts the NSS features and the local information entropy features of the known distorted image in the wavelet domain, and constructs the corresponding feature matrix. Two ABNNs are trained as the image distortion classifier and the image quality scorer by using the known distorted image feature matrix, the distortion types and the subjective scores. The NSS features $\boldsymbol{F}_{1}$ and the local information entropy features $\boldsymbol{F}_{2}$ of the to-be-evaluated distorted images are then extracted in the same way. The constructed feature matrix of the to-be-evaluated distorted image is then entered the trained ABNNs to derive the distortion type and the objective score. The flow chart of the WABNN method is shown in Fig. 9. 
Table 2 Meaning of local information entropy feature vector elements in wavelet domain

\begin{tabular}{cc}
\hline Vector & Meaning \\
elements & Mean $\mu_{w}$ of sub-band local information entropy in the first scale and the horizontal, vertical and diagonal orientations \\
\hline$f_{19}-f_{21}$ & Mean $\mu_{w}$ of sub-band local information entropy in the second scale and the horizontal, vertical and diagonal orientations \\
$f_{22}-f_{24}$ & Mean $\mu_{w}$ of sub-band local information entropy in the third scale and the horizontal, vertical and diagonal orientations \\
$f_{25}-f_{27}$ & Skewness $S_{w}$ of sub-band local information entropy in the first scale and the horizontal, vertical and diagonal orientations \\
$f_{28}-f_{30}$ & Skewness $S_{w}$ of sub-band local information entropy in the second scale and the horizontal, vertical and diagonal orientations \\
$f_{31}-f_{33}$ & Skewness $S_{w}$ of sub-band local information entropy in the third scale and the horizontal, vertical and diagonal orientations \\
$f_{34}-f_{36}$ &
\end{tabular}

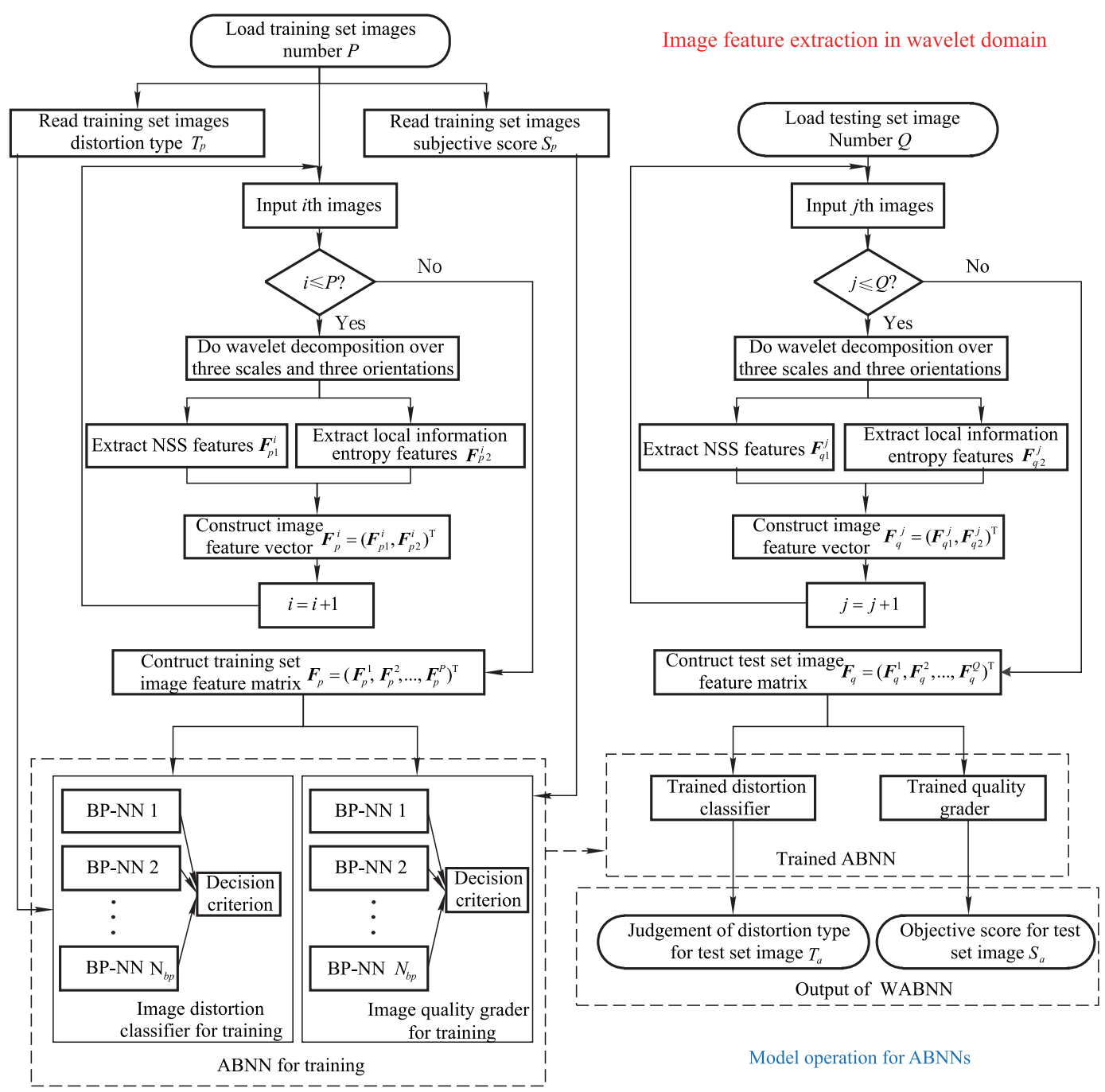

Fig. 9 Flow chart of WABNN method

\subsection{WABNN method implementation steps}

The NR-IQA WABNN method is realized by the following steps:

Step 1 All the distorted images in the database are divided into the training set and the test set. The number of distorted images in the training set is $P$. The number of distorted images in the test set is $Q . P$ and $Q$ are numbers satisfying $P \geqslant 2 Q$.

Step 2 All the distorted images in the training set are applied with wavelet decomposition over three scales and three orientations. NSS features and local information entropy features of each image are extracted according to the methods in Section 2.1 and Section 2.2. Combine these two kinds of features to construct a 36-dimensional image feature vector $\boldsymbol{F}_{p}, \boldsymbol{F}_{p}=\left(\boldsymbol{F}_{p 1}, \boldsymbol{F}_{p 2}\right)^{\mathrm{T}}$, the feature vectors of all training images constitute an image feature matrix $\boldsymbol{F}_{p}$, $\boldsymbol{F}_{p}=\left(\boldsymbol{F}_{p}^{1}, \boldsymbol{F}_{p}^{2}, \ldots, \boldsymbol{F}_{p}^{P}\right)^{\mathrm{T}}$. The size of $\boldsymbol{F}_{p}$ is $p \times 36$.

Step 3 Using the image feature matrix $\boldsymbol{F}_{p}$, the corre- 
sponding subjective scores $S_{p}$ of the training set distorted image, and the corresponding image distortion type $T_{p}$ to train the ABNNs. $\boldsymbol{F}_{p}$ and $S_{p}$ are used to train the neural network grader, and $\boldsymbol{F}_{p}$ and $T_{p}$ are used to train the neural network classifier.

Step 4 The feature vector $\boldsymbol{F}_{q}$ of each distorted image in the test set are extracted by the same method given in Step 2, and the feature matrix $\boldsymbol{F}_{q}$ of the test set images is constructed, $\boldsymbol{F}_{q}=\left(\boldsymbol{F}_{q}^{1}, \boldsymbol{F}_{q}^{2}, \ldots, \boldsymbol{F}_{q}^{Q}\right)^{\mathrm{T}}$, the size of $\boldsymbol{F}_{q}$ is $q \times 36$.

Step 5 The image feature matrix $\boldsymbol{F}_{q}$ of the test set is entered into the trained ABNN to obtain the objective quality score $S_{a}$ and the distortion type judgment $T_{a}$ of the test set image.

\section{2 $\quad \mathrm{ABNN}$ image quality grader and image distortion classifier}

In this paper, two independent ABNNs with the same structure are designed separately as the image quality grader and the image distortion classifier, which are independent of each other, as shown in Fig. 9.

A single ABNN is mainly composed of $N_{b p}$ BP neural networks and a set of preconditions. Each BP neural network works as a weak classifier or weak grader that produces an output. The output of each BP neural network is compared with the preconditions and the results will affect the weight of the BP neural network. Finally, the final output of a single AdaBoost_BP network is calculated as the weighted sum of the outputs of every BP neural network.

\subsubsection{ABNN image quality grader}

The AdaBoost algorithm is an improved self-adaptive boosting algorithm [30]. The idea is to constantly add a new weak classifier until a predetermined small enough error rate is reached [31]. The AdaBoost algorithm is commonly used for classification. In recent years, it has also been used in the field of predictive data analysis and has shown excellent predictive accuracy $[32,33]$.

In the proposed method WABNN, the ABNN is used as an image quality scorer. Specific steps are as follows:

Step 1 Initialize the distribution weights of the training image feature matrix $\boldsymbol{F}_{p}$. Since the size of $\boldsymbol{F}_{p}$ is $p \times 36$, which is composed of the 36-dimensional feature vectors of $P$ distorted images, the initial weight of each distorted image, i.e., the initial distribution weight of training image feature matrix $\boldsymbol{F}_{p}$, is set as follows:

$$
D_{1}(i)=1 / P, \quad i=1, \ldots, P .
$$

Step 2 Initialize each BP neural network in the ABNN scorer. According to the size of the training image feature matrix $\boldsymbol{F}_{p}$, which is $p \times 36$, a total of 36 columns are put in columns. The output of the image scorer is a concrete numerical value. Therefore, each BP neural network is set to be a three-layer structure of $36-n_{0}-1$, where $n_{0}$ is the number of the hidden layer nodes, and $n_{0}=36$ in the experiment. The sigmoid function and the linear function are selected as transfer functions in turn.

Step $3 \quad N_{b p}$ BP neural networks cycle. Let $n=$ $1, \ldots, N_{b p}$ :

(i) The training image feature matrix $\boldsymbol{F}_{p}$ and the training image subjective score $S_{p}$ (the size of $S_{p}$ is $p \times 1$ ) are used as the input and the output of the network to train the $n$th BP neural network. The trained $n$th BP network is denoted as net $_{n}$.

(ii) Calculate the weight $h_{n}$ of the trained $n$th BP network net $_{n}$ :

$$
h_{n}=\frac{1}{2 \times \exp \left|\sum_{i=1}^{P} D_{n}(i)\right|} .
$$

(iii) Put the test image feature matrix $\boldsymbol{F}_{q}$ (the size of $\boldsymbol{F}_{q}$ is $q \times 36$ ) into the network net $t_{n}$ to obtain the output $a_{n}$ of $n e t_{n}$ of the test images. The size of $a_{n}$ is $q \times 1$.

(iv) Put the training image feature matrix $\boldsymbol{F}_{p}$ into the network net $t_{n}$ again to obtain the output $b_{n}$ of net ${ }_{n}$ of the training images. The size of $b_{n}$ is $p \times 1$. Calculate the error between $b_{n}$ and the true subjective scores $S_{p}$ of the training images:

$$
\operatorname{err}_{n}(i)=b_{n}(i)-S_{p}(i), \quad i=1,2, \ldots P .
$$

(v) Calculate the distribution weights of the training image feature matrix $\boldsymbol{F}_{p}$ of the $(n+1)$ th BP neural network:

$$
D_{n+1}(i)=\left\{\begin{array}{l}
k_{0} D_{n}(i), \quad\left|\operatorname{err}_{n}(i)\right|>0.25 \\
D_{n}(i), \quad \text { else }
\end{array}\right.
$$

where $i=1,2, \ldots, P, k_{0}$ is the distribution weight adjustment coefficient, which generally takes value of 1.1. $\left|e r r_{n}(i)\right|>0.25$ is the preset judgment condition, which is determined after many experiments.

(vi) Normalize the distribution weights of training image feature matrices $\boldsymbol{F}_{p}$ in the $(n+1)$ th BP neural network:

$$
D_{n+1}(i)=\frac{D_{n+1}(i)}{\sum_{i=1}^{P} D_{n+1}(i)} .
$$

The purpose of the normalization is to make the sum of the distribution weights of the training image feature matrix to be 1 when the proportion is constant.

Step 4 Determine the output of the ABNN image quality scorer. Firstly, normalize the weights $h_{n}$ of $N_{b p}$ BP neural networks. Then, calculate the weighted sum of $a_{n}$ 
and $h_{n}$. Finally, the output $S_{a}$ of the ABNN image quality scorer is obtained.

$$
\begin{array}{r}
h_{n}=\frac{h_{n}}{\sum_{n=1}^{N_{b p}} h_{n}} \\
S_{a}=\sum_{n=1}^{N_{b p}} a_{n} h_{n}
\end{array}
$$

Repeat the above algorithm several times. The number $N_{b p}$ of BP neural network is set as $N_{b p}=20$.

\subsubsection{ABNN image distortion classifier}

In the method of WABNN, the algorithm of the ABNN image distortion classifier is basically the same as the algorithm of ABNN image quality grader mentioned above with only changes in the form of the input and output. The input of ABNN image distortion classifier includes training the set distorted image feature matrix $\boldsymbol{F}_{p}$ and training the image distortion type $\boldsymbol{T}_{p}$. Keep the form $\boldsymbol{F}_{p}$ unchanged. Since the selected LIVE database contains five types of distortion, $\boldsymbol{T}_{p}$ is set as a vector of the form $p \times 1$. For each element $T_{p}(i) \in\{1,2,3,4,5\}$ in the vector, let 1 represents WN, 2 represents BLUR, 3 represents JPEG, 4 represents JP2K, and 5 represents FF.

The output $T_{a}$ of the ABNN image distortion classifier is obtained by using the algorithm mentioned in Section 3.2.1 with $\boldsymbol{F}_{p}$ and $\boldsymbol{T}_{p}$ as the input. At this point $T_{a}$ is usually a non-integer value. Each value in $T_{a}$ represents a type of image distortion.

$$
T_{a}= \begin{cases}1, & T_{a}<1.5 \\ 2, & 1.5 \leqslant T_{a}<2.5 \\ 3, & 2.5 \leqslant T_{a}<3.5 \\ 4, & 3.5 \leqslant T_{a}<4.5 \\ 5, & T_{a} \geqslant 4.5\end{cases}
$$

\section{Experimental results and analysis}

The proposed WABNN method and the eight NR-IQA methods are performed in the LIVE database and the TID2013 database [34]. Their performance is compared by four indexes, which are root mean-squared error (RMSE), linear pearson correlation coefficient (LPCC), spearman rank order correlation coefficient (SROCC) and kendall rank order correlation coefficient (KROCC). All experiments are performed on a Dell OptiPlex desktop computer, which has an Intel Core i7-4790 processor with $8 \mathrm{G}$ memory. The operating system is Win10, and the experimental platform is Matlab R2014a.

\subsection{Experiments on LIVE database}

\subsubsection{Assessment database preprocessing}

There are 982 images in the LIVE database, including 29 different reference images and 779 distorted images. The distortion types include WN, BLUR, JPEG, JP2K and FF, and the subjective DMOS of distorted images are given as well. In this paper, only 779 distorted images in the LIVE database are selected for training and testing the neural networks. The selected images are divided into five datasets, each dataset contains five or six image classes of different types of distortion and degrees of distortion. The detailed

\begin{tabular}{|c|c|c|c|c|c|c|c|}
\hline \multirow{2}{*}{ Dataset } & \multirow{2}{*}{ Categories of distorted images } & \multicolumn{6}{|c|}{ Types and numbers of distorted image } \\
\hline & & WN & BLUR & JPEG & JP2K & FF & All \\
\hline 1 & $\begin{array}{l}\text { Bikes, building } 2 \text {, buildings, } \\
\text { caps, carnivaldolls, cemetry }\end{array}$ & 30 & 30 & 38 & 34 & 30 & 162 \\
\hline 2 & $\begin{array}{c}\text { Churchandcapitol, lighthouse, dancers, } \\
\text { coinsinfountain, house, loweronih35 }\end{array}$ & 30 & 30 & 36 & 37 & 30 & 163 \\
\hline 3 & $\begin{array}{l}\text { Lighthouse } 2 \text {, manfishing, monarch, } \\
\text { ocean, parrots, paintedhouse }\end{array}$ & 30 & 30 & 37 & 34 & 30 & 161 \\
\hline 4 & $\begin{array}{c}\text { Plane, rapids, sailing1, } \\
\text { sailing2, sailing3, sailing4 }\end{array}$ & 30 & 30 & 34 & 35 & 30 & 159 \\
\hline 5 & $\begin{array}{l}\text { Statue, stream, womanhat, } \\
\text { studentsculpture, woman }\end{array}$ & 25 & 25 & 30 & 29 & 25 & 134 \\
\hline \multicolumn{2}{|c|}{ All distorted images in LIVE database } & 145 & 145 & 175 & 169 & 145 & 779 \\
\hline
\end{tabular}
information of these datasets is shown in Table 3.

Table 3 LIVE database classification

\subsubsection{Subjective consistency of WABNN}

Five-fold cross validation is applied to the above five datasets. Each dataset is used as the test set in turn, while the other four are used as the training set. The WABNN scores $S_{a}$ and the subjective scores $S_{q}$ of the test set images are regressed to obtain the subjective consistency value of the WABNN. At the same time, the distortion type $T_{a}$ of the WABNN method is compared with the true distortion type $T_{q}$ of the test set images to obtain the accuracy of the distortion type judgment of the WABNN.

As shown in Fig. 10, two or three different kinds of 
distorted images corresponding to the same image are selected from the five data sets. The objective score and the type of distortion determined by the WABNN method are compared with the given true distortion type and the
DMOS of each distorted image. Fig. 10 shows that the WABNN method can accurately evaluate the degree of distortion and determine the type of distortion on each dataset, and the objective and subjective scores are consistent.

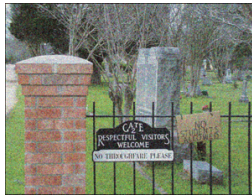

(a) Church and capitol - WN DMOS $=64.03$ $\mathrm{WABNN}=61.09 T_{a}=1$

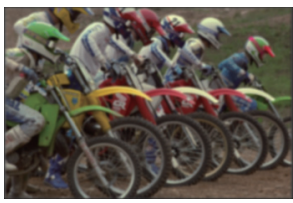

(e) Bikes - BLUR DMOS $=59.73$ $\mathrm{WABNN}=63.59 T_{a}=2$

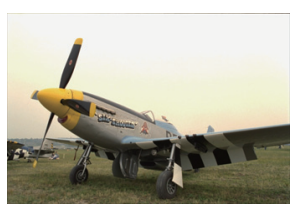

(i) Plane - BLUR DMOS $=18.64$ WABNN $=21.23 \quad T_{a}=2$

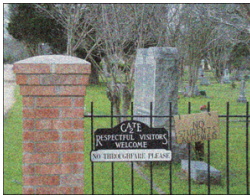

(b) Church and capitol - JPEG DMOS $=72.32$ $\mathrm{WABNN}=75.44 T_{a}=3$

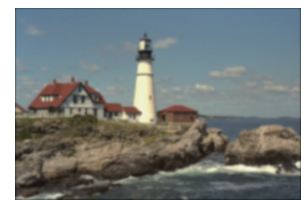

(f) Light house $2-$ BLUR DMOS $=70.26$ $\mathrm{WABNN}=76.69 T_{a}=2$

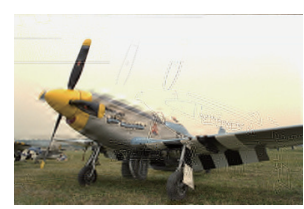

(j) Plane - FF DMOS $=76.03$

$\mathrm{WABNN}=73.47 T_{a}=5$

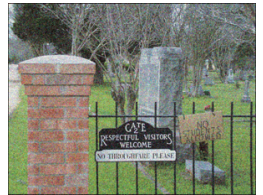

(c) Church and capitol - JP2K DMOS $=25.72$ WABNN $=28.58 \quad T_{a}=4$

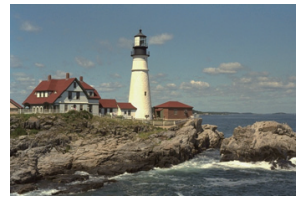

(g) Light house2 - JPEG DMOS $=5.31$

WABNN $=7.32 \quad T_{a}=3$

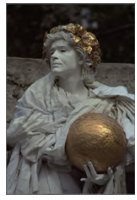

(k) Statue - JP2K DMOS $=36.65$ $\mathrm{WABNN}=40.94 T_{a}=4$

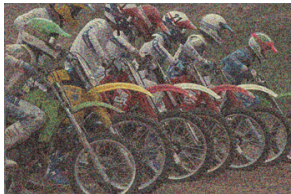

(d) Bikes - WN

DMOS $=74.63$

$\mathrm{WABNN}=75.11 T_{a}=1$

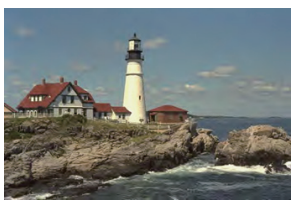

(h) Light house 2 - JP2K DMOS $=52.09$ $\mathrm{WABNN}=48.22 \quad T_{a}=4$

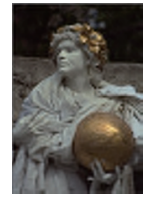

(1) Statue - FF

DMOS $=72.20$ $\mathrm{WABNN}=69.01 \quad T_{a}=5$

Fig. 10 Subjective consistency of WABNN method for different datasets images

Table 4 shows the consistency scores between the objective and subjective scores of the WABNN method for all distorted images on each dataset.

Table 4 Subjective consistency between objective and subjective scores of the WABNN method on different datasets

\begin{tabular}{ccccc}
\hline Dataset & RMSE & LPCC & SROCC & KROCC \\
\hline Dataset 1 & 8.6741 & 0.9484 & 0.9408 & 0.7801 \\
Dataset 2 & 9.1359 & 0.9227 & 0.9173 & 0.7635 \\
Dataset 3 & 9.9486 & 0.9159 & 0.9088 & 0.7562 \\
Dataset 4 & 8.8254 & 0.9355 & 0.9265 & 0.7687 \\
Dataset 5 & 8.9366 & 0.9491 & 0.9352 & 0.7781 \\
\hline All & 9.1107 & 0.9364 & 0.9249 & 0.7664 \\
\hline
\end{tabular}

The results show that the value of the RMSE is lower than 10, the value of LPCC and SROCC are around 0.93, and the value of KROCC is more than 0.75 , which indicates that the WABNN method can accurately evaluate the degree of image distortion without a REF image. The stability and monotonicity of objective scores are good and the results are consistent with the human eye's subjective judgment.

Table 5 shows the accuracy scores of the WABNN method for determining the type of image distortion with- out a reference image for different datasets. The experimental results show that the WABNN method is more than 90\% accurate in determining the type of image distortion on the whole LIVE database, which demonstrates that the WABNN method performs well in classifying and judging the type of image distortion. Comparison of accuracy scores across each distortion type shows that the WABNN method is more accurate in identifying WN, BLUR and JPEG compression (accuracy scores are all higher than $90 \%$ ). The judgment accuracy for WN images is the highest.

Table 5 Accuracy of the distortion type judgment of the WABNN method on different datasets

\begin{tabular}{ccccccc}
\hline Dataset & WN & BLUR & JPEG & JPEG2000 & FF & All \\
\hline Dataset 1 & 96.67 & 90.00 & 97.37 & 85.29 & 86.67 & 91.36 \\
Dataset 2 & 96.67 & 93.33 & 94.44 & 89.19 & 86.67 & 92.02 \\
Dataset 3 & 100.00 & 90.00 & 91.89 & 88.24 & 90.00 & 91.93 \\
Dataset 4 & 100.00 & 93.33 & 88.24 & 88.57 & 86.67 & 91.19 \\
Dataset 5 & 96.00 & 92.00 & 96.30 & 89.66 & 88.00 & 93.13 \\
\hline All & 97.87 & 91.73 & 93.65 & 88.19 & 87.60 & 91.93 \\
\hline
\end{tabular}

Table 4 and Table 5 show that the proposed WABNN method can effectively evaluate the quality of the distorted 
images and distinguish the types of image distortions. Experimental results show a high evaluation accuracy and subjective consistency.

\subsubsection{Comparison of subjective consistency with other NR-IQA methods}

The subjective consistency scores of the eight currently commonly used NR-IQA methods (BIQI, DIIVINE, BLIINDS-II, NIQE, BRISQUE, SSEQ, BHOD, NRSL) for the images of five different distortion types in the LIVE database are shown in Table 6.

Table 6 Comparison of the subjective consistency of different NRIQA methods on LIVE database

\begin{tabular}{|c|c|c|c|c|c|}
\hline $\begin{array}{l}\text { Distortion } \\
\text { type }\end{array}$ & Method & RMSE & LPCC & SROCC & KROCC \\
\hline \multirow{9}{*}{ WN } & BIQI & 6.7214 & 0.9538 & 0.9510 & 0.8219 \\
\hline & DIIVINE & 5.4815 & 0.9880 & 0.9840 & 0.8782 \\
\hline & BLIINDS-II & 6.0112 & 0.9799 & 0.9783 & 0.8511 \\
\hline & NIQE & 6.2546 & 0.9773 & 0.9662 & 0.8495 \\
\hline & BRISQUE & 5.3271 & 0.9851 & 0.9786 & 0.8763 \\
\hline & SSEQ & 5.9289 & 0.9806 & 0.9784 & 0.8597 \\
\hline & BHOD & 6.5544 & 0.9801 & 0.9728 & 0.8645 \\
\hline & NRSL & 5.3846 & 0.9868 & 0.9801 & 0.8916 \\
\hline & WABNN & 5.4394 & 0.9855 & 0.9816 & 0.8708 \\
\hline \multirow{9}{*}{ BLUR } & BIQI & 8.9648 & 0.8293 & 0.8463 & 0.7795 \\
\hline & DIIVINE & 7.9961 & 0.9230 & 0.9210 & 0.7961 \\
\hline & BLIINDS-II & 7.6863 & 0.9381 & 0.9432 & 0.8328 \\
\hline & NIQE & 7.0567 & 0.9525 & 0.9341 & 0.8286 \\
\hline & BRISQUE & 7.2395 & 0.9506 & 0.9435 & 0.8379 \\
\hline & SSEQ & 6.8649 & 0.9607 & 0.9483 & 0.8461 \\
\hline & BHOD & 6.1669 & 0.9660 & 0.9649 & 0.8547 \\
\hline & NRSL & 7.8504 & 0.9447 & 0.9420 & 0.8081 \\
\hline & WABNN & 6.6339 & 0.9572 & 0.9442 & 0.8407 \\
\hline \multirow{9}{*}{ JPEG } & BIQI & 10.1440 & 0.9011 & 0.8914 & 0.7839 \\
\hline & DIIVINE & 9.5101 & 0.9210 & 0.9100 & 0.8085 \\
\hline & BLIINDS-II & 8.6541 & 0.9376 & 0.9311 & 0.8257 \\
\hline & NIQE & 8.2982 & 0.9414 & 0.9382 & 0.8218 \\
\hline & BRISQUE & 7.2042 & 0.9734 & 0.9647 & 0.8466 \\
\hline & SSEQ & 7.5705 & 0.9702 & 0.9510 & 0.8415 \\
\hline & BHOD & 8.1735 & 0.9726 & 0.9461 & 0.8206 \\
\hline & NRSL & 7.5684 & 0.9764 & 0.9508 & 0.8294 \\
\hline & WABNN & 7.4838 & 0.9423 & 0.9348 & 0.8381 \\
\hline \multirow{9}{*}{ JP2K } & BIQI & 12.0133 & 0.8086 & 0.7995 & 0.7368 \\
\hline & DIIVINE & 9.6109 & 0.9220 & 0.9130 & 0.7656 \\
\hline & BLIINDS-II & 9.5055 & 0.9348 & 0.9506 & 0.8168 \\
\hline & NIQE & 9.5024 & 0.9370 & 0.9172 & 0.7818 \\
\hline & BRISQUE & 9.8752 & 0.9229 & 0.9139 & 0.7693 \\
\hline & SSEQ & 8.1653 & 0.9464 & 0.9420 & 0.8011 \\
\hline & BHOD & 7.4821 & 0.9662 & 0.9462 & 0.8180 \\
\hline & NRSL & 7.3111 & 0.9671 & 0.9514 & 0.8243 \\
\hline & WABNN & 8.9165 & 0.9412 & 0.9310 & 0.7951 \\
\hline \multirow{9}{*}{ FF } & BIQI & 18.1032 & 0.7328 & 0.7067 & 0.6064 \\
\hline & DIIVINE & 14.4295 & 0.8880 & 0.8630 & 0.6686 \\
\hline & BLIINDS-II & 13.5055 & 0.8955 & 0.8657 & 0.6841 \\
\hline & NIQE & 12.6058 & 0.9128 & 0.8594 & 0.6653 \\
\hline & BRISQUE & 11.7996 & 0.9148 & 0.8861 & 0.7216 \\
\hline & SSEQ & 12.6858 & 0.9162 & 0.9035 & 0.7493 \\
\hline & BHOD & 10.1813 & 0.9445 & 0.9198 & 0.7760 \\
\hline & NRSL & 11.9348 & 0.9238 & 0.9029 & 0.7535 \\
\hline & WABNN & 11.5913 & 0.9189 & 0.9110 & 0.7662 \\
\hline
\end{tabular}

The scores for the WABNN method are compared with those of the eight existing NR-IQA methods. In Table 6, the bold font indicates that the correlation coefficient of the method is in the top three among all methods.

Table 6 shows that the proposed WABNN method and the existing BHOD and NRSL methods have a high subjective consistency. The proposed WABNN method has 14 out of 20 scores in the top three of the four correlation coefficients for five different types of distorted images, while BHOD and NRSL each has 13 out of 20 scores in the top three, indicating that the proposed WABNN method achieves good evaluation results for all types of distortion and thus exhibits high robustness.

The scatter plots of the subjective and objective consistency scores of different NR-IQA methods are shown in Fig. 11.

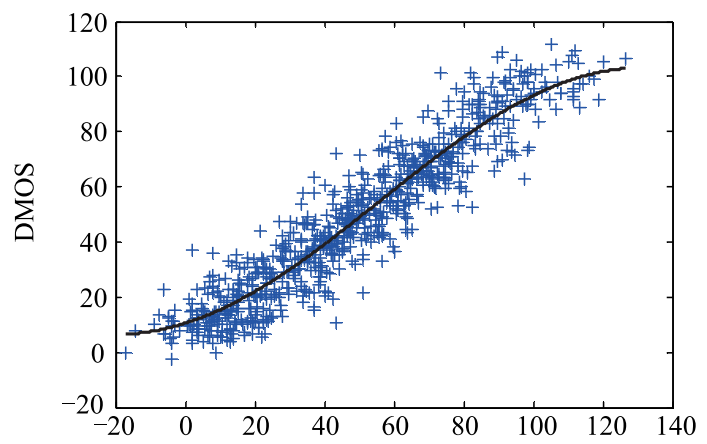

(a) Objective score by WABNN

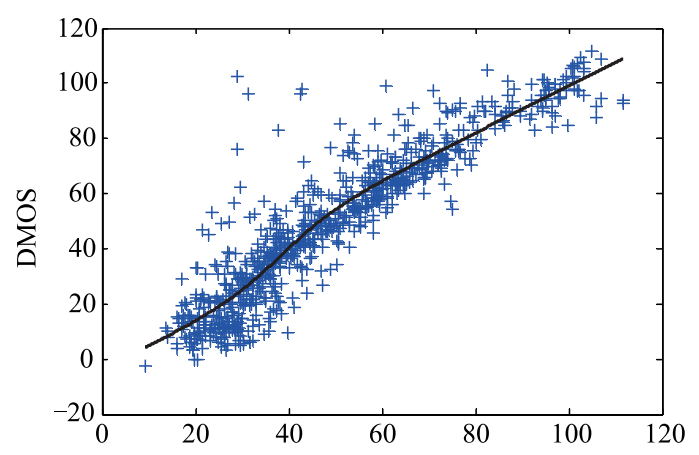

(b) Objective score by SSEQ

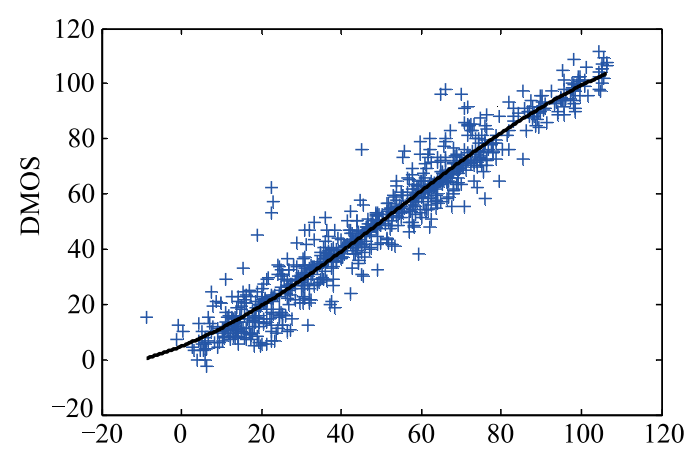

(c) Objective score by BRISQUE 


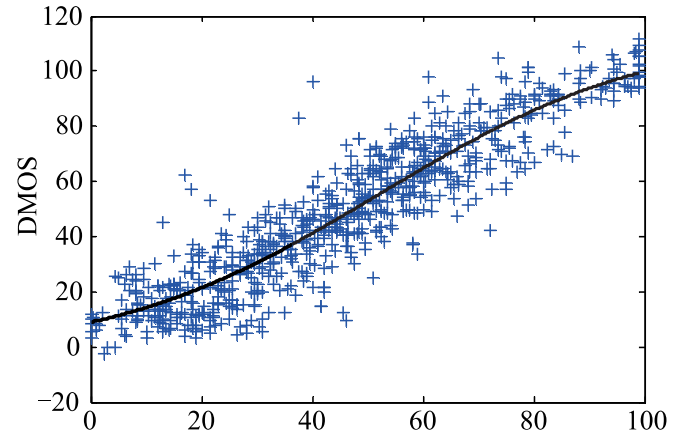

(d) Objective score by BLIINDS-II

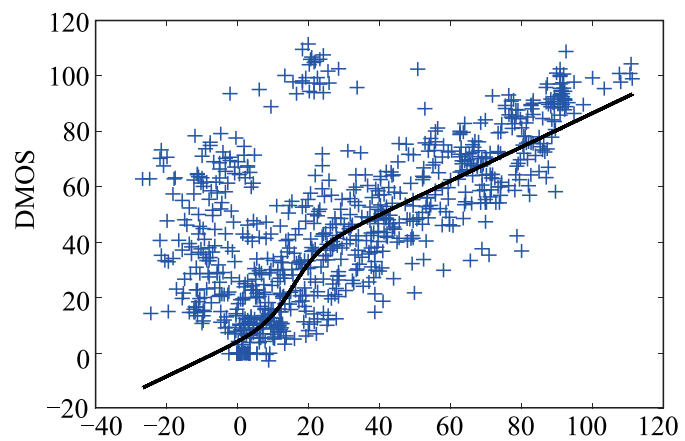

(e) Objective score by BRISQUE

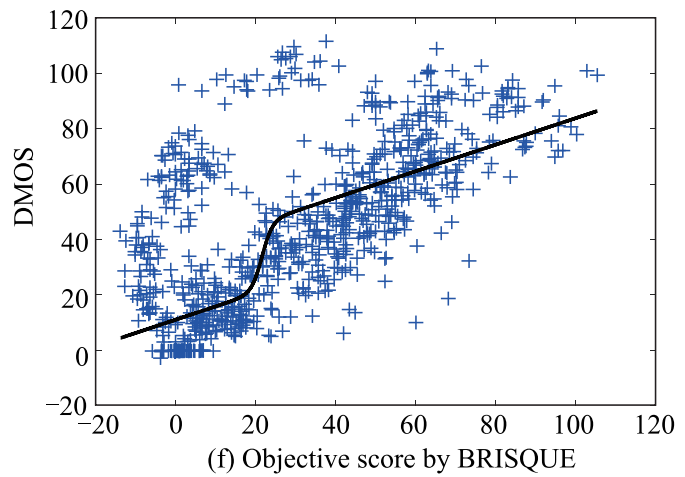

+ : Images in LIVE; - - Curve fitted with logistic function.

Fig. 11 Scatter plots of the subjective and objective consistency scores of different NQ-IQA methods

The $x$-axis denotes the objective score obtained by the IQA method and the $y$-axis denotes the subjective score obtained by human eyes. Fig. 11 shows that, compared with BHOD and other methods, the scatter points of WABNN, SSEQ and BRISQUE are more concentrated and the deviation of the points from the fitting curves is smaller, indicating a better objective-subjective consistency.

Table 6 and Fig. 11 show that, compared with other methods which can only give the quality score of the distorted image, the proposed WABNN method not only gives quality scores with high subjective consistency, but also can determine the type of image distortion, indicating the superiority of the proposed WABNN method over the existing methods.

\subsection{Experiments on TID2013 database}

\subsubsection{Comparison of subjective consistency with other NR-IQA methods}

The 24 natural images in TID2013 database are selected and the distortion types include WN, BLUR, JPEG and JP2K. The subjective consistency scores of the eight currently commonly used NR-IQA methods for the images with four different distortion types in the TID2013 database are shown in Table 7. The scores for the WABNN method are compared with those of the eight existing NRIQA methods. In Table 7, the bold font indicates that the correlation coefficient of the method is in the top three among all methods.

Table 7 Comparison of the subjective consistency of different NRIQA methods on TID2013 database

\begin{tabular}{|c|c|c|c|c|c|}
\hline $\begin{array}{c}\text { Distortion } \\
\text { type }\end{array}$ & Method & RMSE & LPCC & SROCC & KROCC \\
\hline \multirow{9}{*}{ WN } & BIQI & 0.2922 & 0.9106 & 0.9015 & 0.7377 \\
\hline & DIIVINE & 0.3383 & 0.8773 & 0.8104 & 0.7089 \\
\hline & BLIINDS-II & 0.3857 & 0.8395 & 0.8215 & 0.6267 \\
\hline & NIQE & 0.3994 & 0.8428 & 0.8155 & 0.6050 \\
\hline & BRISQUE & 0.3305 & 0.8841 & 0.8688 & 0.6833 \\
\hline & SSEQ & 0.3108 & 0.9002 & 0.8946 & 0.7267 \\
\hline & BHOD & 0.4858 & 0.7295 & 0.7106 & 0.5221 \\
\hline & NRSL & 0.3127 & 0.8967 & 0.8891 & 0.7133 \\
\hline & WABNN & 0.2804 & 0.9163 & 0.9166 & 0.7289 \\
\hline \multirow{9}{*}{ BLUR } & BIQI & 0.6356 & 0.8552 & 0.8362 & 0.6633 \\
\hline & DIIVINE & 0.4799 & 0.9211 & 0.9165 & 0.7600 \\
\hline & BLIINDS-II & 0.3803 & 0.9510 & 0.9462 & 0.8114 \\
\hline & NIQE & 0.6702 & 0.8254 & 0.8155 & 0.5890 \\
\hline & BRISQUE & 0.4837 & 0.9194 & 0.9115 & 0.7490 \\
\hline & SSEQ & 0.5312 & 0.9012 & 0.8931 & 0.7200 \\
\hline & BHOD & 0.6682 & 0.9193 & 0.9107 & 0.7467 \\
\hline & NRSL & 0.3965 & 0.9456 & 0.9346 & 0.7933 \\
\hline & WABNN & 0.4303 & 0.9345 & 0.9287 & 0.7796 \\
\hline \multirow{9}{*}{ JPEG } & BIQI & 1.0220 & 0.7288 & 0.6703 & 0.4933 \\
\hline & DIIVINE & 0.6845 & 0.8844 & 0.8104 & 0.6200 \\
\hline & BLIINDS-II & 0.4615 & 0.9507 & 0.9054 & 0.7330 \\
\hline & NIQE & 0.5595 & 0.9268 & 0.8665 & 0.6498 \\
\hline & BRISQUE & 0.5212 & 0.9361 & 0.8708 & 0.7000 \\
\hline & SSEQ & 0.4622 & 0.9487 & 0.9009 & 0.7379 \\
\hline & BHOD & 0.4753 & 0.9483 & 0.8712 & 0.6867 \\
\hline & NRSL & 0.4134 & 0.9587 & 0.9005 & 0.7323 \\
\hline & WABNN & 0.4139 & 0.9446 & 0.9198 & 0.7663 \\
\hline \multirow{9}{*}{ JP2K } & BIQI & 0.7612 & 0.8934 & 0.8220 & 0.6311 \\
\hline & DIIVINE & 0.6367 & 0.9249 & 0.8773 & 0.7000 \\
\hline & BLIINDS-II & 0.5466 & 0.9449 & 0.9269 & 0.7800 \\
\hline & NIQE & 0.7155 & 0.9072 & 0.8981 & 0.7062 \\
\hline & BRISQUE & 0.6723 & 0.9128 & 0.9073 & 0.7523 \\
\hline & SSEQ & 0.6011 & 0.9328 & 0.8999 & 0.7379 \\
\hline & BHOD & 0.6093 & 0.9332 & 0.8907 & 0.7200 \\
\hline & NRSL & 0.6562 & 0.9204 & 0.8831 & 0.7133 \\
\hline & WABNN & 0.6996 & 0.9136 & 0.9066 & 0.7493 \\
\hline
\end{tabular}

Table 7 shows that the proposed WABNN method and 
the existing BLIINDS-II and SSEQ methods have a high subjective consistency. The proposed WABNN method has 13 out of 16 scores in the top three of the four correlation coefficients for four different types of distorted images, while BLIINDS-II and SSEQ have 12 and 8 out of 16 scores in the top three respectively, indicating that the proposed WABNN method achieves good evaluation results for all types of distortion and thus exhibits high robustness.

\subsubsection{Accuracy of the distortion type judgment of the WABNN method}

Table 8 shows the accuracy scores of the WABNN method for determining the type of image distortion without the REF image. The experimental results show that the accuracy of the WABNN method in determining the type of image distortion on the selected four distorted databases of the TID2013 is 79\%, which demonstrates that the WABNN method performs well in classifying and judging the type of image distortion. Comparison of the accuracy scores across each distortion type shows that the WABNN method is more accurate in identifying WN and JPEG compression (accuracy scores are all higher than $80 \%$ ). The judgment accuracy for WN images is the highest.

Table 8 Accuracy of the distortion type judgment using the WABNN method

\begin{tabular}{cccccc}
\hline & WN & BLUR & JPEG & JP2K & ALL \\
\hline accuracy & 88 & 72 & 80 & 76 & 79 \\
\hline
\end{tabular}

\subsection{Database independence experiments}

To verify that the performance of the WABNN is independent of the particular database used, database independence experiments are performed on the LIVE database and the TID2013 database. $80 \%$ of the distorted images in the LIVE database are selected as the training set, and $80 \%$ of the distorted images in the TID2013 database are selected as the test set. The SROCC are used as the testing indicator. The subjective consistency scores of the eight currently commonly used NR-IQA methods for the images of four different distortion types are shown in Table 9. Furthermore, $80 \%$ of the distorted images in the TID2013 database are selected as the training set, and $80 \%$ of the distorted images in the LIVE database are selected as the test set. The SROCC are used as the testing indicator. The subjective consistency scores of the eight NR-IQA methods for the images of four different distortion types are shown in Table 10. The scores for the WABNN method are compared with those of the eight existing NR-IQA methods.

Table 9 Comparison of the subjective consistency of different NRIQA methods on LIVE database (training set) and TID2013 database (test set)

\begin{tabular}{cccccc}
\hline Method & WN & Blur & JPEG & JP2K & ALL \\
\hline BIQI & 0.8768 & 0.8766 & 0.6665 & 0.8559 & 0.8190 \\
DIIVINE & 0.8839 & 0.8939 & 0.8524 & 0.7895 & 0.8549 \\
BLIINDS-II & 0.7231 & 0.5833 & 0.5662 & 0.6112 & 0.6210 \\
NIQE & 0.8155 & 0.8155 & 0.8665 & 0.8981 & 0.8489 \\
BRISQUE & 0.9067 & 0.9045 & 0.8917 & 0.8888 & $\mathbf{0 . 8 9 7 9}$ \\
SSEQ & 0.8616 & 0.8986 & 0.9183 & 0.9023 & $\mathbf{0 . 8 9 5 2}$ \\
BHOD & 0.7489 & 0.9148 & 0.8815 & 0.9155 & 0.8652 \\
NRSL & 0.8422 & 0.9094 & 0.9092 & 0.7779 & 0.8597 \\
WABNN & 0.9010 & 0.8971 & 0.9221 & 0.8412 & $\mathbf{0 . 8 9 0 4}$ \\
\hline
\end{tabular}

Table 10 Comparison of the subjective consistency of different NRIQA methods on LIVE database (test set) and TID2013 database (training set)

\begin{tabular}{cccccc}
\hline Method & WN & Blur & JPEG & JP2K & ALL \\
\hline BIQI & 0.6562 & 0.8483 & 0.5995 & 0.7592 & 0.7158 \\
DIIVINE & 0.4061 & 0.9237 & 0.9237 & 0.8482 & 0.7754 \\
BLIINDS-II & 0.9479 & 0.8455 & 0.8697 & 0.7010 & 0.8410 \\
NIQE & 0.9662 & 0.9341 & 0.9382 & 0.9172 & $\mathbf{0 . 9 3 8 ~ 9}$ \\
BRISQUE & 0.8976 & 0.8734 & 0.9570 & 0.8974 & 0.9064 \\
SSEQ & 0.6098 & 0.8810 & 0.9495 & 0.9222 & 0.8406 \\
BHOD & 0.9444 & 0.9036 & 0.9236 & 0.9273 & $\mathbf{0 . 9 2 4 7}$ \\
NRSL & 0.9701 & 0.8408 & 0.9355 & 0.9300 & $\mathbf{0 . 9 1 9 1}$ \\
WABNN & 0.9580 & 0.8472 & 0.9484 & 0.8984 & $\mathbf{0 . 9 1 3 0}$ \\
\hline
\end{tabular}

Table 9 and Table 10 show that the SROCC of the proposed WABNN method is about 0.9 , indicating that the method has database independence.

\subsection{Time consumption of WABNN}

The mean time that the seven good performance NRIQA methods (DIIVINE, BLIINDS-II, SSEQ, BRISQUE, BHOD, NRSL, WABNN) spent to extract features from all images in the LIVE database and the TID2013 database are shown in Table 11 and Table 12, respectively. The runtime of the NR-IQA methods is mainly generated in the process of extracting image features.

Table 11 Mean time of extracting all images features for different NR-IQA methods on LIVE database

\begin{tabular}{ccccccccc}
\hline & DIIVINE & BLIINDS-II & SSEQ & BRISQUE & BHOD & NRSL & WABNN \\
\hline Mean time/s & 19.6450 & 62.3720 & 2.1357 & 0.1718 & 0.8273 & 0.2964 & 0.2513 \\
\hline & Table 12 & Mean time of extracting all images features for different NR-IQA methods on TID2013 database & \\
\hline & DIIVINE & BLIINDS-II & SSEQ & BRISQUE & BHOD & NRSL & WABNN \\
\hline Mean time/s & 14.7338 & 62.3720 & 1.4146 & 0.1117 & 0.5498 & 0.1703 & 0.2386 \\
\hline
\end{tabular}


Table 11 and Table 12 show that the mean time of the feature extraction of the proposed WABNN method is fewer than that of DIIVINE, BLIINDS-II, SSEQ and BHOD methods both in the LIVE database and the TID2013 database. For the NRSL method, the mean time is $0.0451 \mathrm{~s}$ more in LIVE and merely $0.0683 \mathrm{~s}$ fewer in TID2013 than that of the WABNN. Compared with the BRISQUE method, the mean time is merely $0.0795 \mathrm{~s}$ fewer in LIVE and $0.1269 \mathrm{~s}$ fewer in TID2013 than that of the WABNN. Therefore, the proposed WABNN method has a high assessment accuracy and operation efficiency.

\section{Conclusions}

In this paper, a 36-dimensional image feature vector consists of NSS features and two-dimensional wavelet-domain local information entropy features in three scales. Two ABNNs are trained as the image distortion classifier and the image quality scorer by using a known distorted image feature matrix, a distortion type and the subjective scores. A series of contrast experiments are carried out in the LIVE database and TID2013 database. Experimental results show the high accuracy of the distinguishing distortion type, the high consistency with subjective scores and the high robustness of the proposed method for distorted images. Moreover, experimental results show the independence for the databases and the relatively high operation efficiency. The research mainly focuses on the single-distorted images. Assessment of multiply-distorted images, which is of greater practical significance, will be addressed in future research.

\section{References}

[1] MANAP R A, SHAO L. Non-distortion-specific no-reference image quality assessment: a survey. Information Sciences, 2015, 301(C): $141-160$.

[2] ZHU X, MILANFAR P. A no-reference sharpness metric sensitive to blur and noise. Proc. of International Workshop on Quality of Multimedia Experience, 2009: 64-69.

[3] CHEN M J, BOVIK A C. No-reference image blur assessment using multiscale gradient. Proc. of International Workshop on Quality of Multimedia Experience, 2009: 1-11.

[4] MARICHAL X, MA W Y, ZHANG H J. Blur determination in the compressed domain using DCT information. Proc. of the IEEE International Conference on Image Processing, 2002, 12: $386-390$

[5] SHAO Y, SUN F, LI H. No-reference remote sensing image quality assessment method using visual properties. Journal of Tsinghua University, 2013, 53(4): 550-555. (in Chinese)

[6] WANG Z, BOVIK A C, EVAN B L. Blind measurement of blocking artifacts in images. Proc. of the IEEE International Conference on Image Processing, 2000: 981 - 984.

[7] SAZZAD Z M P, KAWAYOKE Y, HORITA Y. No reference image quality assessment for JP2K based on spatial features. Signal Processing Image Communication, 2008, 23(4): $257-$ 268.

[8] HORITA Y, ARATA S, MURAI T. No-reference image quali- ty assessment for JPEG/JP2K coding. Proc. of the IEEE Signal Processing Conference, 2004: 1301-1304.

[9] XIE F, PAN X, JIANG Z, et al. No-reference haze effects assessment for remote sensing images. Chinese Journal of Stereology \& Image Analysis, 2015, 20(1): 22-27. (in Chinese)

[10] WANG Z, BOVIK A C. Modern image quality assessment. New York: Morgan and Claypool Publishing Company, 2006.

[11] MOORTHY A K, BOVIK A C. A two-step framework for constructing blind image quality indices. IEEE Signal Processing Letters, 2010, 17(5): $513-516$.

[12] LI C, BOVIK A C, WU X. Blind image quality assessment using a general regression neural network. IEEE Trans. on Neural Networks, 2011, 22(5): $793-799$.

[13] MOORTHY A K, BOVIK A C. Blind image quality assessment: from natural scene statistics to perceptual quality. IEEE Trans. on Image Processing: A Publication of the IEEE Signal Processing Society, 2011, 20(12): 3350-3364.

[14] SAAD M A, BOVIK A C, CHARRIER C. DCT statistics model-based blind image quality assessment. Proc. of the IEEE 18th International Conference on Image Processing, 2011, 9: $3154-3157$.

[15] SAAD M A, BOVIK A C, CHARRIER C. Blind image quality assessment: a natural scene statistics approach in the DCT domain. IEEE Trans. on Image Processing: A Publication of the IEEE Signal Processing Society, 2012, 21(8): 3339-3352.

[16] MITTAL A, SOUNDARARAJAN R, BOVIK A C. Making a "completely blind" image quality analyzer. IEEE Signal Processing Letters, 2013, 20(3): 209-212.

[17] MITTAL A, MOORTHY A K, BOVIK A C. No-reference image quality assessment in the spatial domain. IEEE Trans. on Image Processing: A Publication of the IEEE Signal Processing Society, 2012, 21(12): $4695-4708$.

[18] MITTAL A, MOORTHY A K, BOVIK A C. Blind/referenceless image spatial quality evaluator. Proc. of the IEEE 45th Asilornar Conference on Signals, Systems and Computers, 2011: $723-727$.

[19] LIU L, LIU B, HUANG H, et al. No-reference image quality assessment based on spatial and spectral entropies. Signal Processing Image Communication, 2014, 29(8): 856-863.

[20] LI Q, LIN W, FANG Y. No-reference image quality assessment based on high order derivatives. Proc. of the IEEE International Conference on Multimedia and Expo, 2016: 1-6.

[21] LI Q, LIN W, XU J, et al. Blind image quality assessment using statistical structural and luminance features. IEEE Trans. on Multimedia, 2016, 18(12): 2457 - 2469.

[22] LIU L, HUA Y, ZHAO Q, et al. Blind image quality assessment by relative gradient statistics and adaboosting neural network. Signal Processing Image Communication, 2016, 40(C): $1-15$.

[23] RUDERMAN D L. The statistics of natural images. Network Computation in Neural Systems, 2009, 5(4): 517-548.

[24] WANG Z, SIMONCELLI E P, BOVIK A C. Multiscale structural similarity for image quality assessment. Proc. of the 37 th IEEE Asilomar Conference on Signals, Systems \& Computers, 2003, 2: 1398 - 1402 .

[25] SRIVASTAVA A, LEE A B, SIMONCELLI E P, et al. On advances in statistical modeling of natural images. Journal of Mathematical Imaging \& Vision, 2003, 18(1): 17-33.

[26] SHEIKH H R, WANG Z, BOVIK A C, et al. Image and video quality assessment research at LIVE 2004. http://live.ece.utexas.edu/research/quality.

[27] SHEIKH H R, SABIR M F, BOVIK A C. A statistical evaluation of recent full reference image quality assessment algorithms. IEEE Trans. on Image Processing, 2006, 15(11): 
$3440-3451$.

[28] SHARIFI K, LEON-GARCIA A. Estimation of shape parameter for generalized Gaussian distributions in subband decompositions of video. IEEE Trans. on Circuits \& Systems for Video Technology, 1995, 5(1): 52-56.

[29] ZHOU Y L, CHEN F, SUN B. Identification method of gas-liquid two-phase flow pattern based on image wavelet packet information entropy and genetic neural network. Nuclear Power Engineering, 2008, 29(1): 115 - 120. (in Chinese)

[30] ZHANG Z. Digital image processing and machine vision. Beijing: The Posts and Telecommunications Press, 2010. (in Chinese)

[31] CAO Y, MIAO Q G, LIU J C, et al. Advance and prospects of adaboost algorithm. Acta Automatica Sinica, 2013, 39(6): $745-758$. (in Chinese)

[32] WU J L, ZHANG B H, WANG K. Application of Adaboostbased BP neural network for short-term wind speed forecast. Power System Technology, 2012, 36(9): 221-225.

[33] LI S, XIE Y L, WANG W X. Application of AdaBoost_BP neural network in prediction of railway freight volumes.Computer Engineering and Applications, 2012, 48(6): 233-234. (in Chinese)

[34] PONOMA N, PONOMARENKO N, JIN L, et al. Image database TID2013: peculiarities, results and perspectives. Signal Processing: Image Communication, 2015, 30: 57-77.

\section{Biographies}

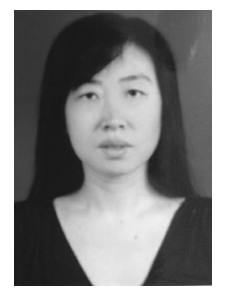

YAN Junhua was born in 1972. She received her B.S. degree, M.S. degree and Ph.D. degree from Nanjing University of Aeronautics and Astronautics in 1993, 2001 and 2004, respectively. She is a professor at Nanjing University of Aeronautics and Astronautics. Her research interests include image quality assessment, multi-source information fusion, target detection, tracking and recognition.

E-mail: yjh9758@126.com

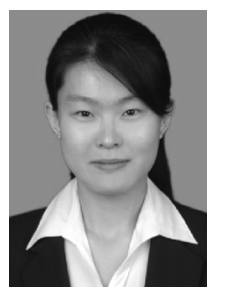

BAI Xuehan was born in 1993. She received her B.S. degree from Harbin Institute of Technology in 2016. She is a graduate student at Nanjing University of Aeronautics and Astronautics. Her research interest is image quality assessment.

E-mail: 1595720931@qq.com

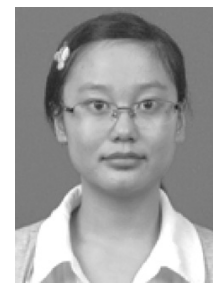

ZHANG Wanyi was born in 1992. She received her B.S. degree and M.S. degree from Nanjing University of Aeronautics and Astronautics in 2014 and 2017. Her research interest is image quality assessment.

E-mail: daisyzwy917@126.com

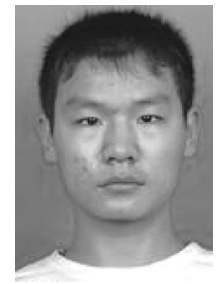

XIAO Yongqi was born in 1994. He received his B.S. degree and M.S. degree from Nanjing University of Aeronautics and Astronautics in 2015 and 2018. His research interest is image quality assessment.

E-mail: couragexyq@163.com

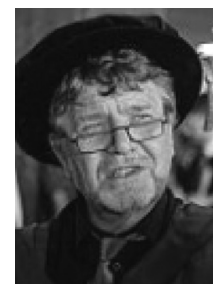

CHATWIN Chris was born in 1950. He received his B.S. degree from University of Aston in 1973, his M.S. and Ph.D. degrees from University of Birmingham in 1977, and 1980, respectively. He is a professor in Engineering at University of Sussex. His research interests are smart cameras and algorithms, UAV surveillance systems, multi camera tracking systems, image processing for cancer diagnostics. E-mail: C.R.Chatwin@sussex.ac.uk

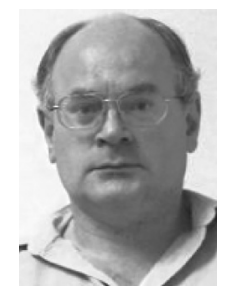

YOUNG Rupert was born in 1959. He received his B.S. and Ph.D. degrees from University of Glasgow in 1986, and 1994, respectively. He is a reader at University of Sussex. His current research interests are machine vision, image processing, neural networks, and quantum computing.

E-mail: R.C.D.Young@sussex.ac.uk

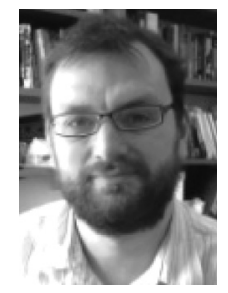

BIRCH Phil was born in 1973. He received his B.S., M.S. degree and Ph.D. degrees from University of Durham in 1994, and 1999, respectively. He is a senior lecturer at University of Sussex. His current research interests are optical signal processing, target detection and computer vision. E-mail:p.m.birch@sussex.ac.uk 